\title{
Developing an evidence-based clinical pathway for the assessment, diagnosis and management of acute Charcot Neuro-Arthropathy: a systematic review
}

Tamara E Milne ${ }^{1 *}$, Joseph R Rogers ${ }^{2}$, Ewan M Kinnear ${ }^{3}$, Helen V Martin $^{3}$, Peter A Lazzarini ${ }^{4,5}$, Thomas R Quinton ${ }^{6}$ and Frances M Boyle ${ }^{7}$

\begin{abstract}
Background: Charcot Neuro-Arthropathy (CN) is one of the more devastating complications of diabetes. To the best of the authors' knowledge, it appears that no clinical tools based on a systematic review of existing literature have been developed to manage acute CN. Thus, the aim of this paper was to systematically review existing literature and develop an evidence-based clinical pathway for the assessment, diagnosis and management of acute $\mathrm{CN}$ in patients with diabetes.

Methods: Electronic databases (Medline, PubMed, CINAHL, Embase and Cochrane Library), reference lists, and relevant key websites were systematically searched for literature discussing the assessment, diagnosis and/or management of acute CN published between 2002-2012. At least two independent investigators then quality rated and graded the evidence of each included paper. Consistent recommendations emanating from the included papers were then fashioned in a clinical pathway.

Results: The systematic search identified 267 manuscripts, of which 117 (44\%) met the inclusion criteria for this study. Most manuscripts discussing the assessment, diagnosis and/or management of acute CN constituted level IV (case series) or EO (expert opinion) evidence. The included literature was used to develop an evidence-based clinical pathway for the assessment, investigations, diagnosis and management of acute $\mathrm{CN}$.

Conclusions: This research has assisted in developing a comprehensive, evidence-based clinical pathway to promote consistent and optimal practice in the assessment, diagnosis and management of acute CN. The pathway aims to support health professionals in making early diagnosis and providing appropriate immediate management of acute $\mathrm{CN}$, ultimately reducing its associated complications such as amputations and hospitalisations.
\end{abstract}

Keywords: Charcot Neuro-Arthropathy, Management, Clinical pathway, Diabetes

\section{Background}

Charcot Neuro-Arthropathy $(\mathrm{CN})$ is one of the more devastating complications affecting patients with diabetes and peripheral neuropathy [1]. It is a progressive, destructive condition that is characterised by acute fractures, dislocations and joint destruction in the weight-bearing neuropathic foot [2]. The acute phase is often misdiagnosed and can rapidly lead to severe foot deformity, ulceration and

\footnotetext{
* Correspondence: Tamara_Milne@health.qld.gov.au

'Podiatry Department, Ipswich General Hospital, Brisbane, Australia Full list of author information is available at the end of the article
}

amputation [1,3,4]. Early diagnosis and management of acute $\mathrm{CN}$ is therefore imperative to avoid the rapid progression towards permanent foot deformation and its associated complications [5].

There are many reported aetiologies of $\mathrm{CN}$, however in modern western societies diabetes mellitus has become the leading cause [1,5-7]. The true prevalence of $\mathrm{CN}$ is unknown, most likely due to a high incidence of mistaken or delayed initial diagnosis [7], but a number of population-based studies have reported an estimated prevalence of $0.4-13 \%$ in patients with diabetes $[7,8]$.

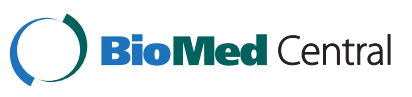


To date, best practice assessment, diagnosis and management of acute $\mathrm{CN}$ appears to be influenced more by expert consensus than a rigorous evidence-base $[1,5,6]$. This may be because acute $\mathrm{CN}$ is considered one of the more rare complications of those caused by diabetes and thus tends to fall outside of the existing national guidelines or systematic reviews on diabetic foot complications [9]. This paper therefore aims to systematically review current relevant literature and develop an evidence-based clinical pathway for the assessment, diagnosis and management of acute $\mathrm{CN}$ in patients with diabetes.

\section{Methods}

\section{Search strategy}

A systematic review of the most relevant $\mathrm{CN}$ literature published between 2002-2012 was undertaken in the process of developing the clinical pathway. The search strategy was designed to identify relevant literature that focussed on the clinical assessment, diagnosis and/or conservative management of acute $\mathrm{CN}$. For the purpose of this study the terms Charcot, Arthropathy, Neuroarthropathy, Osteoarthropathy, Neuro-Osteoarthropathy, and Neurogenic-Arthropathy were used interchangeably. The subsequent clinical pathway was guided by the recommendations specified by the National Health and Medical Research Council of Australia (NHMRC), 1999 [10,11].

Electronic databases (Medline, PubMed, CINAHL, Embase and Cochrane Library: Databases of Systematic Reviews) were searched for relevant literature by the first author in August 2012. Key search terms used were Charcot, Arthropathy, Neuroarthropathy, Osteoarthropathy, Neuro-Osteoarthropathy, and Neurogenic-Arthropathy. The search strategies for each database are summarised in Additional file 1. The exclusion criteria included papers published prior to 2002, not written in English, non-diabetes papers, or papers discussing the surgical management only of acute $\mathrm{CN}$. As the focus of this paper was providing a contemporary clinical pathway for nonsurgical health professionals, the authors considered the last decade of publications to be appropriate and to exclude surgical papers.

The initial search was intentionally broad in order to identify all literature pertaining to $\mathrm{CN}$, and thus included both empirical evidence and expert opinion. To ensure completeness, the first author hand searched the reference lists of the initial manuscripts identified, searched web pages of relevant diabetes organisations for clinical practice guidelines, and contacted local and international experts in the field in an effort to identify any literature that may not have been identified in the initial search.

\section{Study selection}

All titles and abstracts retrieved by the initial search were scanned by the first author using the following screening question: Does the article discuss the clinical assessment, diagnosis and/or conservative management of acute $C N$ in the diabetic foot? If the article was deemed to meet the screening question, the first author retrieved the full text for quality assessment by the co-authors.

\section{Quality assessment}

Co-authors, with expertise in diabetes related foot complications (TM, JR, EK, HM, PL, TQ), reviewed all identified full texts. At least two blinded co-authors independently reviewed each included article to assess its relevance and quality, and grade its level of evidence according to the NHMRC guidelines [9-11]. Table 1 provides definitions for the NHMRC levels of evidence $[9,10]$. Firstly, co-authors were required to review the full text to ensure it met all original inclusion criteria and to specifically exclude articles that focussed only on surgical management of $\mathrm{CN}$ or $\mathrm{CN}$ in non-diabetes populations. Secondly, co-authors were asked to assess if the article was of adequate quality or methodologically sound. In consideration of the small amount of literature published on $\mathrm{CN}$, the definition of methodologically sound was broadened to exclude only articles not reporting methods or procedures (for example letters to the editor or commentaries). Lastly, the co-authors rated the article to assign it a level of evidence according to NHMRC guidelines [10,11]. Any inconsistencies between the assessments of manuscripts were resolved by the assessment of a third co-author.

\section{Data extraction}

Literature that met the final inclusion criteria was then used to construct the clinical pathway. In an attempt to aid clinical management, the authors decided to base the development and flow of the pathway on the clinical phases evident in current general clinical management. These phases include assessments, investigations, diagnosis and management. Any common recommendations emanating from the final literature search were identified by the first and second authors and entered into the clinical domains. Clinical recommendations on the pathway were also welcomed from experts where quality evidence was lacking. The recommendations were prioritised according to level of evidence and relevance to the clinical pathway (Additional file 2, Additional file 3, Additional file 4). The final pathway was agreed to by the consensus of all co-authors.

\section{Results}

A total of 267 manuscripts were identified from the initial search strategy. Of these, 117 (44\%) were assessed to meet the final inclusion criteria and were used in the development of the clinical pathway. The 150 (56\%) articles excluded were either considered lacking in quality 
Table 1 NHMRC levels of evidence

\begin{tabular}{ll}
\hline $\begin{array}{l}\text { Level of } \\
\text { evidence }\end{array}$ & Definition \\
\hline II & A systematic review of level II studies \\
III & A randomised controlled trial \\
III-2 & A pseudorandomised controlled trial (i.e. alternate allocation or some other method) \\
III-3 & A comparative study with concurrent controls (i.e. non-randomised experimental trial, cohort study, case-control study) \\
IV & A comparative study without concurrent controls (i.e. historical cohort study, two or more single arm study) \\
EO & Case series \\
& $\begin{array}{l}\text { Expert opinion - where evidence was absent or unreliable and advice was formulated based on the clinical judgement and } \\
\text { experience of experts in the field }\end{array}$
\end{tabular}

or did not meet the final inclusion criteria. The large majority of manuscripts included were either expert opinion (67.5\%) or level IV evidence (19\%). Only three level II randomised control studies (RCT's) were identified. Table 2 summarises the evidence levels of all included manuscripts. Table 3 summarises the country of publication origin of all included manuscripts. Most manuscripts were published in either the USA (50\%) or UK (26\%).

The pathway is divided into the four key phases for the clinical management of acute $\mathrm{CN}$ that have been addressed by the included literature. These phases are 1) Assessment, 2) Investigations, 3) Diagnosis, and 4) Management of acute $\mathrm{CN}$. Unfortunately, some areas pertaining to these phases of management are devoid of quality research and in these instances low-level evidence, such as expert opinion, was utilised. The clinical pathway is presented in Figure 1.

\section{Assessment}

\section{Clinical signs \& symptoms}

Localised unilateral swelling, erythema, warmth, +/pain $(50 \%),+/$ - deformity: level of evidence $=$ IV It is well reported that acute $\mathrm{CN}$ characteristically presents with localised swelling, erythema and increased temperature $\left(>2^{\circ} \mathrm{C}\right.$ compared to the contralateral foot) to the affected foot $[1,3,12,13]$. Owing to the presence of peripheral neuropathy, pain may not always be present (reportedly in only $50 \%$ of cases) or will be less than expected given the severity of the clinical findings $[12,14,15]$. The diagnosis of acute $\mathrm{CN}$ is primarily dependant on this initial clinical presentation and therefore

Table 2 Evidence levels of included manuscripts

\begin{tabular}{ll}
\hline Level of evidence & Included manuscripts (117) \\
\hline II & $0.0 \%(0)$ \\
III & $2.5 \%(3)$ \\
IV & $11.0 \%(13)$ \\
EO & $19.0 \%(22)$ \\
\hline
\end{tabular}

requires high clinical suspicion by the treating clinician for all patients with diabetes and peripheral neuropathy who present with these clinical signs and symptoms [14]. More advanced presentations of acute CN may also present with obvious foot deformity, including the characteristic 'rocker-bottom' deformity that is emblematic of $\mathrm{CN}$ [16].

Urgent referral to multidisciplinary high risk foot service: level of evidence $=\mathrm{EO}$ If acute $\mathrm{CN}$ is suspected, an urgent referral to a multidisciplinary high-risk foot service or specialist clinic is recommended for appropriate multidisciplinary management of this complex condition $[9,11,12,17,18]$.

\section{Clinical assessments}

History of trauma (25-50\%): level of evidence = III-2 or recent surgery: level of evidence $=$ IV Preceding trauma may be recalled in as many as half of all cases of acute $\mathrm{CN}(25-50 \%)[3,15,16,19-22]$. The role of trauma

Table 3 Country of publication of included manuscripts

\begin{tabular}{ll}
\hline Country of publication & Included manuscripts (117) \\
\hline USA & $50 \%(58)$ \\
UK & $26 \%(31)$ \\
Germany & $7 \%(8)$ \\
Netherlands & $6 \%(7)$ \\
Israel & $2 \%(2)$ \\
Scandinavia & $2 \%(2)$ \\
Australia & $1 \%(1)$ \\
Canada & $1 \%(1)$ \\
China & $1 \%(1)$ \\
France & $1 \%(1)$ \\
Hong Kong & $1 \%(1)$ \\
India & $1 \%(1)$ \\
Morocco & $1 \%(1)$ \\
Sweden & $1 \%(1)$ \\
Switzerland & $1 \%(1)$
\end{tabular}




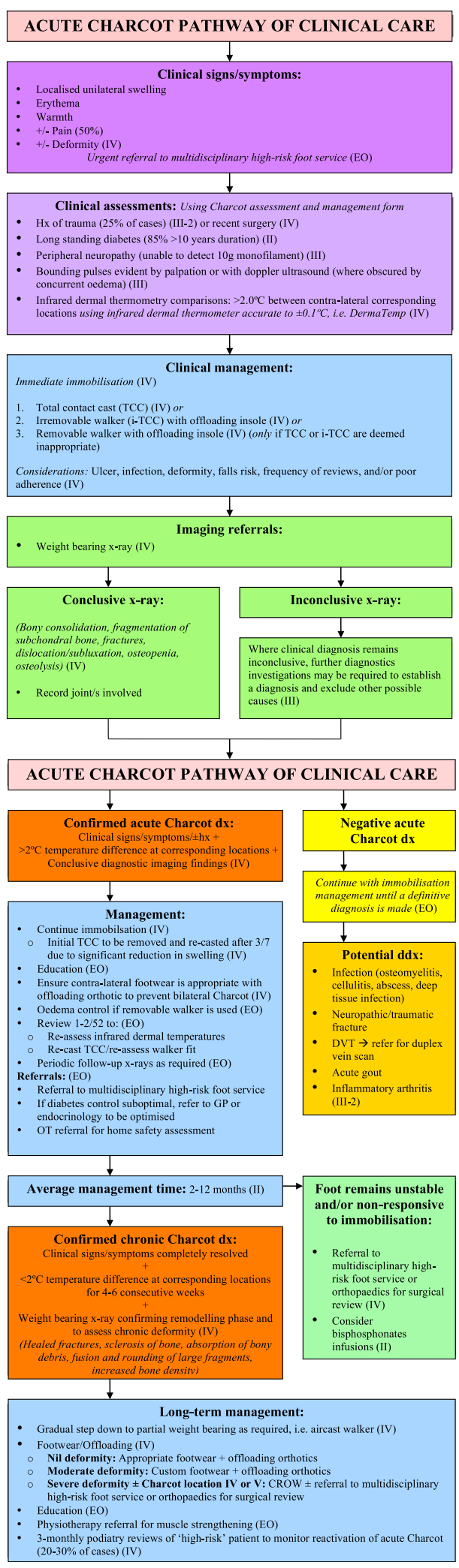

Figure 1 Acute Charcot Pathway of Clinical Care. in an insensate extremity has been reported as an important factor in the pathogenesis of acute $\mathrm{CN}$ and should therefore be queried at the initial presentation $[15,21]$. However, due to the presence of an insensate extremity, it is important to consider recall bias as a cofounding factor and therefore a history of trauma may be unreliable [1]. In incidences where no trauma is recalled, repetitive micro-trauma on an insensate foot may be a contributing factor $[9,21,22]$.

Recent foot surgery has also been described as a possible precipitating factor to acute $\mathrm{CN}[3,23]$. The precise mechanisms by which surgery affects the pathogenesis of $\mathrm{CN}$ remain unclear, however it is reported that it may be associated with the local inflammation following surgery or alternatively as a result of the foot deformity following pedal amputation [1,24]. Pedal amputation can functionally compromise the foot leading to altered weight bearing forces that result in repetitive micro-trauma, a reported precipitating factor of acute CN $[25,26]$.

Long-standing diabetes: level of evidence $=$ II The relationship between duration of diabetes and the onset of acute $\mathrm{CN}$ is well reported in a number of clinical trials and case series. Most commonly, at the time of onset patients with both Type 1 or Type 2 diabetes have been diagnosed for a period $>10$ years $[8,13,27-30]$.

Peripheral neuropathy: level of evidence $=$ III The presence of peripheral sensory neuropathy is an important component for the onset of acute $\mathrm{CN}$ with no reported cases developing in its absence $[1,8,31,32]$. Peripheral sensory neuropathy can be accurately assessed using the Semmes-Weinstein 10 g monofilament [8,9,11,16,32].

Normal peripheral arterial perfusion: level of evidenceIII Generally, the acute $\mathrm{CN}$ foot has well preserved arterial perfusion. Pedal pulses can be palpated and are often described as 'bounding' in the acute $\mathrm{CN}$ foot, unless obscured by associated swelling. In this instance, the use of a doppler ultrasound may be required to assess arterial perfusion $[1,8,9,11,31]$.

Infrared dermal thermometry comparisons $>2^{\circ} \mathrm{C}$ : level of evidence $=$ IV Given the local inflammatory response during the acute phase of $\mathrm{CN}$, temperature monitoring with the use of a handheld infrared dermal thermometre is a useful diagnostic assessment tool $[33,34]$. Infrared dermal thermometry comparisons between contralateral corresponding locations are typically $>2.0^{\circ} \mathrm{C}$ in the affected foot $[21,35,36]$. Temperatures should be assessed approximately 15 minutes after the cast and footwear is removed and the use of an infrared dermal thermometer precise to $\pm 0.1^{\circ} \mathrm{C}$ for a more 
accurate assessment is recommended [37]. Due to an absence of studies objectively determining or comparing different sites for temperature assessment, recommendations vary amongst the literature. Most frequently, however, skin temperatures are measured at the following 9 sites: dorsal mid foot, hallux, medial 1st metatarsal head, plantar 3rd metatarsal head, lateral 5th metatarsal head, 1st metatarsal-cuneiform joint, talonavicular joint, cuboid, plantar heel, and ankle [33,37].

\section{Immediate clinical management}

Immediate immobilisation: level of evidence $=$ IV In order to reduce the risk of severe chronic deformity, if acute $\mathrm{CN}$ is suspected, immediate immobilsation should be implemented until a definitive diagnosis is determined [12,38-40]. Immobilisation remains the cornerstone therapy for acute $\mathrm{CN}$ and is essential to break the cycle of repetitive trauma propagating the acute phase and to ultimately prevent the progression of deformity $[1,41,42]$. Options for immobilsation include the total contact cast and irremovable/removable walkers.

Total contact casts and irremovable walkers: level of evidence $=$ IV Total contact casts $($ TCCs) were originally referred to as the 'gold standard' immobilsation therapy for acute $\mathrm{CN}$, due to their custom and irremovable nature [43-46]. The TCC is a custom moulded cast, commonly using plaster of Paris or fibreglass, which maintains contact with the entire planter service of the foot and lower limb [43-46]. The TCC immobilises the affected foot and ankle, reduces plantar foot pressures and swelling, protects from additional trauma, and maintains patient mobility $[42,47]$.

An alternative to the TCC is the instant total contact cast (iTCC) which has been reported to be just as effective in immobilising the acute $\mathrm{CN}$ foot, as well as being more cost effective and requiring less skill to apply $[1,48]$. An iTCC consists of a prefabricated removable walker that is rendered irremovable by simply applying a layer of tape or fibreglass cast roll around the body of the walker to encourage patient compliance $[2,46]$.

Removable walkers: level of evidence $=$ IV Prefabricated removable cast walkers have the benefit of immediate application without specialist skills and have been reported to be just as effective in offloading the diabetic foot, however patient adherence is often significantly reduced with these devices [31,41,47,48]. A large observational study of 288 patients with acute $\mathrm{CN}$, reported that the use of irremovable offloading (TCC or iTCC) shortened the median time to resolution by approximately 3 months when compared with removable walkers [3]. This study highlights the issue with patient adherence when removable devices are prescribed. As a result, removable walkers should only be prescribed when TCCs or iTCCs are deemed inappropriate.

Previous studies have advocated complete non-weight bearing immobilsation with the use of crutches through the initial acute phase, however it has been reported that a three-point gait may in fact increase the load on the contralateral foot and thereby predispose the patient to bilateral acute $\mathrm{CN}[1,2,43]$. Two recent case series demonstrated that ambulatory casting during the acute phase of $\mathrm{CN}$ does not negatively impact the outcome of $\mathrm{CN}$ and may in fact reduce the loss of muscle tone and bone density during immobilsation $[1,42,44]$. Given the paucity of empirical evidence regarding this issue, it is recommended that protective weight bearing should be advised at the discretion of the treating clinician.

Immobilisation considerations: level of evidence $=$ IV There are a number of important factors to consider before prescribing the most appropriate immobilsation device for the individual patient. The benefits of the TCC may be limited by the need for specially trained clinicians, available clinical time for application and product cost. In addition, cast changes are required within the first 3 days for the initial cast and 1-2 weekly thereafter to maintain proper fit and where necessary, permit wound management [1]. These frequent reviews may be particularly problematic for patients who live in rural or underserviced communities that are distant from specialised diabetic foot care clinics. In contrast, patients with current foot deformity may be at risk of secondary ulceration if fitted to a prefabricated walker and therefore a TCC may be the only appropriate means of immobilsation. Lastly, patients with $\mathrm{CN}$ often have increased instability and are at risk of falls as a result of multiple co-morbidities, including loss of proprioception and postural hypertension, and therefore aggressive cast immobilsation may not be appropriate and alterative modalities may need to be considered, such as a wheelchair $[1,15,47]$.

\section{Investigations} Imaging referrals

Plain weight bearing radiographs: level of evidence $=$ IV If a patient presents with localised unilateral swelling, erythema and increased temperature in an insensate foot, plain radiographs are an important first line investigation and can be invaluable in ascertaining the presence of $\mathrm{CN}$. In most cases, no further imaging studies are required to confirm diagnosis [1,2]. Characteristic radiographic signs of acute $\mathrm{CN}$ include bony consolidation, fragmentation of subchondral bone, fractures, dislocations, subluxations, osteopenia and osteolysis $[35,49,50]$. Although controversial, weight-bearing radiographs without immobilsation can be valuable in identifying 
subtle fractures, fragmentations and joint subluxation seen in very early stages of acute $\mathrm{CN}$, which may not be present on standard non-weight bearing films. Additionally, joint deformity or collapse is often more accurately assessed in weight bearing views, and therefore weightbearing views should be considered at the discretion of the clinician $[21,39,44,50]$.

Where clinical diagnosis remains inconclusive at this time, further diagnostic investigations may be required to establish a diagnosis and exclude other possible causes.

Repeat radiographs at 2 weeks: level of evidence $=\mathrm{EO}$ Normal radiographs at presentation does not necessarily exclude $\mathrm{CN}$ and it therefore may be important to perform further imaging investigations to confirm the diagnosis $[15,49]$. Easily available and inexpensive, repeat radiographs can be a valuable tool in confirming in the final diagnosis, especially in more remote locations where other diagnostic imaging modalities are not available. Repeat $\mathrm{x}$-rays are generally obtained after 2 weeks of the initial investigation as the radiographic signs of acute $\mathrm{CN}$ generally become more conspicuous during this period $[33,34]$.

Magnetic resonance imaging: level of evidence $=$ III Magnetic resonance imaging (MRI) represents a noninvasive and sensitive diagnostic tool in the study of bone marrow and soft tissue abnormalities, providing high quality images of the foot [51]. MRI has the ability to detect subtle changes in the early stages of acute $\mathrm{CN}$, such as bone marrow oedema, before they are evident on plain radiographs $[1,51,52]$. This can play an important role in the early diagnosis of acute $\mathrm{CN}$, when radiographs are inconclusive, thereby improving clinical outcomes [51,52]. MRI has also been reported to be both sensitive (77-100\%) and specific (80\%-100\%) in the diagnosis of $\mathrm{CN}$ and osteomyelitis, a well reported challenge for most treating clinicians $[1,11,49,53]$. Where available and appropriate for use, MRI should be the imaging modality of choice for the diagnosis of acute $\mathrm{CN}$ when radiographs are inconclusive $[1,9,11,33]$.

Nuclear medicine: level of evidence $=$ IV Nuclear medicine includes a number of diagnostic tests based on the use of radioisotopic tracers [1]. Nuclear medicine may play an important role in the diagnosis of acute $\mathrm{CN}$ where other imaging modalities, such as MRI, are contraindicated or unavailable [51]. Three-phase bone scans are highly sensitive $(<100 \%)$ to acute bone pathology but lack specificity for acute CN $[49,51]$. For patients with low clinical suspicion of osteomyelitis and no signs of $\mathrm{CN}$ on initial radiographs, three-phase bone scans have proven to be a useful tool in assisting in the diagnosis.
Alternatively, leukocyte-labelled bone or marrow scans (99 m Tc HMPAO or 111 Indium) provide improved specificity (69-80\%) for distinguishing infection from acute $\mathrm{CN}$ and are a more appropriate imaging tool when underlying infection is suspected $[1,8,18,49]$.

FDG-PET: level of evidence $=$ IV More recently, 18 F-fluorodeoxyglucose positron emission tomography (FDG-PET) has been recognised as having potential for differentiating acute $\mathrm{CN}$ from osteomyelitis $[1,49]$. A few recent studies have reported that combined FDG-PET may have several advantages over existing imaging techniques for diagnosing osteomyelitis and acute $\mathrm{CN}$, including improved sensitivity (100\%) and specificity (93.8\%) for acute $\mathrm{CN}$, high-quality images with detailed anatomic localisation, and fast results within 1.5 to 2 hours after the initiation of the examination [54-56]. However, the 2011 international task force consensus document on the Charcot foot in diabetes recommends that FDG-PET for the potential diagnosis of acute $\mathrm{CN}$ and osteomyelitis remains investigational at this time [1].

Bone biopsy: level of evidence $=$ EO Bone biopsy remains the only diagnostic method for definitive discrimination between osteomyelitis and $\mathrm{CN}$. This is not, however, always appropriate and can potentially result in a number of secondary complications including infection, excessive bleeding, pain, fracture, or new onset of acute $\mathrm{CN}[53,57,58]$. With these limitations in mind, bone biopsy should only performed if the diagnosis remains inconclusive after imaging is exhausted, or if osteomyelitis is likely [53].

\section{Serology referrals}

There is currently no universally accepted serology criterion for the diagnosis of $\mathrm{CN}$; however, some studies have reported that serology markers may assist in narrowing the diagnosis $[8,11,14,15,35,39,59]$.

Leukocytosis, C-reactive protein, and erythrocyte sedimentation rate: level of evidence $=$ IV Leukocytosis (WCC), an elevated C-reactive protein (CRP) and erythrocyte sedimentation rate (ESR), and recent unexplained hyperglycemia are all systemic responses to infection $[11,14,39]$. The utility of these inflammatory parameters for identifying infection has been supported throughout the literature and could therefore be a valuable assessment tool for differentiating between acute $\mathrm{CN}$ and infection $[8,15,35,59]$. Recent studies have demonstrated that there is dissociation between the local and systemic inflammatory response in acute $\mathrm{CN}$, with serum WCC, CRP, and ESR values remaining within the reference range for patients with acute $\mathrm{CN}$ despite the 
presence of local inflammation [35,59]. Therefore, in the absence of elevated systematic inflammatory markers, infection may be an unlikely diagnosis and acute $\mathrm{CN}$ should be considered [21].

Glycosylated haemoglobin: level of evidence $=$ IV Chronic hyperglycaemia is a major incipient factor in the development of $\mathrm{CN}$, a theory well supported by the literature [60]. One recent case study, reported that an elevated glycosylated haemoglobin (HbA1c) is associated with more than a $30 \%$ increase in the risk for developing $\mathrm{CN}$ [61]. As mentioned earlier, recent unexplained hyperglycaemia may also assist in the diagnosis of infection [14]. Clinicians have long recognised the importance of tight glycaemic control in reducing the risk of diabetic foot complications [61]. Therefore, as part of a multidisciplinary approach to the management of a patient with diabetes and suspected $\mathrm{CN}$, it is recommended the HbA1c be assessed and, where necessary, optimised [2].

Electrolytes and renal function: level of evidence $=$ IV Diabetic nephropathy has been reported to be associated with an increased incidence of acute $\mathrm{CN}$ [28]. One case study reported that renal failure nearly doubled the risk of $\mathrm{CN}$ (OR 2.1, $\mathrm{p}<0.001$ ), suggesting that patients with co-existing renal failure be carefully monitored for signs of acute $\mathrm{CN}$ [61].

Calcium and vitamin D: level of evidence = EO Pathological or traumatic fractures are a reported misdiagnosis of acute $\mathrm{CN}$, often associated with deficiencies in calcium and vitamin $\mathrm{D}$ resulting in inadequate mineralisation of the bone $[1,59,62]$. Furthermore, if bisphosphonates are administered as part of the management plan, they require adequate levels of calcium and vitamin D to work effectively $[6,63,64]$. Therefore, investigating calcium and vitamin $\mathrm{D}$ levels may be beneficial in assisting with the diagnosis and/or directing the management plan.

Uric acid: level of evidence $=$ EO An acute gout attack may also masquerade as acute $\mathrm{CN}$, however can be excluded by measurement of serum uric acid, which is typically raised in the presence of gout $[15,39,65]$.

\section{Diagnosis}

\section{Acute CN diagnosis criteria}

Level of evidence $=$ IV In the absence of rigorous evidence, the most commonly accepted criteria by treating clinicians for the diagnosis of acute $\mathrm{CN}$ is: a warm, swollen, erythematic foot (clinical signs), with or without any significant history of trauma or surgery, a temperature difference from the contralateral foot of $>2^{\circ} \mathrm{C}$, and conclusive diagnostic images suggestive of acute $\mathrm{CN}$
$[1,13,29,55]$. In the presence of a wound or history of osteomyelitis, clinical suspicion and assessment of osteomyelitis should be considered $[2,11,12,16]$.

\section{Negative diagnosis}

Level of evidence $=\mathbf{E O}$ In the event that $\mathrm{CN}$ may not be the most likely diagnosis, experts in the field recommend continuing with immobilisation until a definitive diagnosis is made so that the risk of foot deformity or other associated complications can be avoided if in fact $\mathrm{CN}$ is later diagnosed $[4,38]$.

\section{Differential diagnosis}

Level of evidence $=$ III-2 Historically, misdiagnoses for acute $\mathrm{CN}$ have included infection (osteomyelitis, cellulitis, abscess, deep tissue infection), DVT, acute gout, neuropathic/traumatic fractures, sprain, or inflammatory arthritis $[4,17,21,22,59]$. One retrospective case series reported that $80 \%$ of patients with acute $\mathrm{CN}$ were initially misdiagnosed as having sprains $(n=11)$, DVT $(n=3)$, osteomyelitis $(n=4)$, tumour (3), cellulitis $(n=6)$, or rheumatoid arthritis $(n=2)$ [17]. Given its rare presentation, it is not surprising that a large number of cases of acute $\mathrm{CN}$ are initially misdiagnosed; however, this only further emphasises the need for high clinical suspicion when a patient with diabetes and neuropathy presents with the clinical signs and symptoms suggestive of acute $\mathrm{CN}$.

\section{Management}

\section{Acute management}

Continue immobilisation: level of evidence $=$ IV Immobilsation of the affected foot continues until complete resolution of the acute phase $[3,31,42]$. The cast is initially replaced (TCC) or re-fit (iTCC, removable walker) after the first 3 days due to the significant oedema reduction seen after this period. The cast is then replaced 1-2 weekly after this time, again to adjust for limb volume changes from oedema and to assess for any complications secondary to immobilsation $[1,43]$.

Education: level of evidence $=$ EO Patient education regarding the diagnosis, estimated length of treatment and expected outcomes is an important component of $\mathrm{CN}$ management. If the patient understands the nature of this limb-threatening condition, they may be more motivated to adhere to the management plan. Emphasis on the importance of strict immobilisation, attending regular follow-up reviews and optimising glucose control may improve the outcome of $\mathrm{CN}[11,39,58,66]$.

Appropriate contralateral footwear: level of evidence $=$ IV Bilateral $\mathrm{CN}$ is reported in as many as $30 \%$ of cases $[8,45,67]$. As stated earlier, immobilsation therapy, 
especially with the use of crutches, has been reported to potentially increase the load on the contralateral foot and thereby predispose the patient to bilateral acute $\mathrm{CN}$ $[1,2]$. For this reason, prophylactic support with appropriate footwear and accommodative insoles is recommended for the contralateral foot to minimise the risk of bilateral acute $\mathrm{CN}[9,39,68,69]$.

Oedema control: level of evidence $=$ EO When a TCC is applied to immobilise the acute $\mathrm{CN}$, the compression of the cast will assist in reducing the oedema present in the acute phase. However, when a prefabricated walker is used it is recommended that oedema be managed with alternative compression therapies such as elastic bandaging $[31,70]$.

Regular reviews: level of evidence $=$ IV During the immobilisation period, regular reviews by a high-risk foot service are important to monitor the activity of the acute phase, review the management plan, and assess and manage any secondary complications [1]. Measuring skin temperature differences between the affected and the non-affected foot using an infrared dermal thermometre is an objective measure for monitoring reduction in inflammation during the acute phase of $\mathrm{CN}$ $[21,34,66]$. The literature suggests that elevated temperatures will correlate with the location of $\mathrm{CN}$ and that temperatures in the affected foot will decrease as acute $\mathrm{CN}$ progresses into the chronic phase [33]. TCC's should be re-casted and the fit of walkers re-assessed at 1-2 weekly reviews to adjust to limb volume changes as the oedema subsides during immobilisation $[2,43,44,71]$.

Periodic follow-up radiographs: level of evidence $=\mathrm{EO}$ Following the initial diagnosis, follow-up radiographs of the affected foot every 4-6 weeks will monitor the progression of $\mathrm{CN}$, as well as any changes in the architectural alignment and configuration of the foot $[8,33,34,42,70]$. However, given the paucity of empirical evidence recommending the benefit of periodic followup radiographs, these are performed at the discretion of the treating clinician.

Appropriate referrals: level of evidence $=\mathrm{EO}$ Given the complexity of $\mathrm{CN}$, a multidisciplinary approach to the holistic management of the patient is recommended [72]. Where appropriate, the authors recommend referrals to a multidisciplinary high-risk foot clinic $[12,17,39]$, local general practitioner or specialist physician to optimise diabetes management and/or other relevant comorbidities $[61,64,66,73]$, and occupational therapy for a home environment assessment, especially when crutches or a wheel chair are prescribed [15].
Bisphosphonates: level of evidence $=$ II There are currently conflicting reports on the clinical benefits of bisphosphonates for the management of acute $\mathrm{CN}$ [6]. Systematic reviews of clinical trials have indicated that bisphosphonates are ineffective and may even be harmful to the resolution time of the acute phase of $\mathrm{CN}$ $[3,6,13]$. In contrast, other studies of the same level of evidence have supported their use, suggesting that bisphosphonates may improve the resolution time of the acute phase by reducing skin temperature and disease activity [72,74-76]. Therefore, given the inconclusive evidence on their use, it is recommended that bisphosphonates be used at the discretion of the treating physician for cases of acute $\mathrm{CN}$ that are non-responsive to conservative immobilsation management.

Average management time: level of evidence $=$ II A number of clinical trials and case series have reported average management times for the complete resolution of $\mathrm{CN}$ between 2-12 months, with a period of 6 months being most commonly reported $[3,13,17,27,28,31,42,47]$. The literature suggests that the management time may be influenced by the location of $\mathrm{CN}$, type of immobilsation used, and the stage of $\mathrm{CN}$ when immobilisation is implemented $[3,17,20,39,47]$.

\section{Chronic CN diagnosis criteria}

Level of evidence $=$ IV The duration of immobilisation is guided by the clinical assessment that the acute phase has completely resolved [1]. This is evident by the resolution of all clinical signs and symptoms, stabilised contralateral skin temperatures, and evidence of healing on radiographs [13,31]. Previous studies have recommended a skin temperature difference between contralateral locations of $<2^{\circ} \mathrm{C}$ for $2-4$ consecutive weeks before transitioning patients from cast immobilisation to a removable walker or appropriate footwear [21,31-33]. Radiographs are an important tool in assisting in the diagnosis of chronic $\mathrm{CN}$ and are recommended once all clinical signs and symptoms have resolved [49]. Radiographic evidence of chronic $\mathrm{CN}$ includes healed fractures, sclerosis of bone, absorption of bony debris, fusion and rounding of large fragments, and increased bone density $[15,49,68,76]$. Feet with severe $\mathrm{CN}$ deformity are siginificantly associated with midfoot ulceration. Therefore, weight bearing radiographs of chronic $\mathrm{CN}$ may be more beneficial at this time to assess the presence and degree of deformity so that appropriate long term offloading can be prescribed [77].

\section{Long-term management}

Partial weight bearing: level of evidence $=$ IV Once the foot is stable, transition to protected weight bearing is generally advised before the patient steps down to footwear $[2,30,66]$. Aircast walkers or other similar 
prefabricated removable walkers have gained acceptance as useful protective modalities for this initial period of weight bearing $[15,44,66]$. Partial weight bearing has been reported to minimise the risk of reactivation of the acute phase if immobilsation is ceased too early [20,31].

Footwear and offloading: level of evidence = IV Footwear is an important component of the long-term management of the insensate chronic $\mathrm{CN}$ foot, ensuring that it remains accommodated, offloaded and protected.

In patients with nil to minor foot deformity after the resolution of acute $\mathrm{CN}$, prefabricated footwear with extra depth and a stiff rocker bottom walking sole may suffice. These shoes, when fitted with custom-molded, fullcontact insoles, will adequately minimise load bearing and mobility of the foot during walking $[2,8,20,30,44,47]$.

In the presence of moderate deformity, custom-made or modified shoes are generally necessary to accommodate the chronic foot deformity. Again, these shoes should be fitted with custom-molded, full contact insoles to minimise load bearing and mobility during walking $[8,20,78]$.

Chronic $\mathrm{CN}$ that has resulted in severe foot deformities and/or $\mathrm{CN}$ that is located in the ankle or rear foot (location IV or V) can often be difficult to stabilise with footwear and typically requires more aggressive long term management such as a Charcot Restraint Orthotic Walker (CROW) to achieve stability and reduce the risk of reactivating the acute phase $[1,15,40,46]$. The CROW has proven to be useful in maintaining foot and ankle alignment in the instable or surgical corrected $\mathrm{CN}$ foot [78], however, where aggressive conservative management has failed, surgical correction of the deformity should be considered [1].

Education: level of evidence $=$ EO Patient education should form an essential component of the long-term management of these patients, focusing on the importance of appropriate footwear and offloading, regular follow up reviews, and the risk of further complications $[11,12,66]$.

Rehabilitation: level of evidence $=$ EO Following an extended period of immobilsation, there will likely be wasting of the calf muscles, loss of bone density and joint stiffness $[42,44]$. Protective rehabilitation with a physiotherapist is recommended following the transition phase out of immobilisation, being cautious, however, of the risk of reactivation of the acute phase or ulceration of bony deformity by excessive rapid mobilisation during the early stages of rehabilitation [15,37].

Long term follow up and/or reactivation: level of evidence $=$ IV Three monthly podiatry reviews of these high-risk patients is advised to monitor for signs of recurrent or new episodes of $\mathrm{CN}$, as well as any other diabetic foot complications $[1,9,12,15]$. Recurrence is reported in $15-30 \%$ of patients with a previous history of $\mathrm{CN}[3,15,69,79]$.

Surgical: level of evidence $=$ IV Typically, if the correct diagnosis is made in the acute phase of $\mathrm{CN}$ and conservative treatment is successful, surgery may be avoided and the risk of subsequent ulcerations and/or amputation may be decreased [30]. Surgical management is usually only considered in the chronic phase of $\mathrm{CN}$ where joint instability and/or severe deformity have failed to be effectively managed with a conservative approach $[1,2,30]$. Up to $50 \%$ of patients have been reported to undergo surgical procedures for long-term management of $\mathrm{CN}$ deformities and instabilities, most commonly occurring 4 years after the initial acute phase [2,30]. Surgery is generally avoided during the acute phase of $\mathrm{CN}$ due to the risk of mechanical failure or secondary infection [1].

\section{Discussion}

Our systematic search of relevant literature highlights that $\mathrm{CN}$ continues to be a poorly understood disorder of the diabetic foot. Although recent clinical research has improved our level of knowledge regarding its etiology and management, there are still only a few high-level evidence-based studies regarding the assessment, diagnosis and management of acute $\mathrm{CN}$ [39]. As hypothesised, most literature pertaining to this field constitutes level IV or EO evidence and no systematic reviews were identified. Thus, this review begins to fill a gap commonly found in Australian and international diabetic foot complication guidelines that overlook the systematic review of $\mathrm{CN}$ [9].

$\mathrm{CN}$ continues to be a persistent challenge for clinicians, especially in its acute phase [6]. The literature reports that the diagnosis of $\mathrm{CN}$ is missed in as many as $79 \%$ of cases and an accurate diagnosis can be delayed up to 29 weeks. This highlights a clear gap in professional education, which this pathway hopes to address [4,21]. Moreover, it is well reported that patients with $\mathrm{CN}$ experience increased morbidity and mortality, a higher risk of amputations, and a reduced quality of life $[5,6,30]$.

Currently, most available clinical guidelines on the management of acute $\mathrm{CN}$ are without a rigorous evidencebase, as displayed in the current pathway $[2,18,80]$. Therefore in the era of evidence-based medicine, this research has assisted in developing a comprehensive, evidencebased clinical pathway designed to promote consistent and optimal practice in the assessment, diagnosis and management of acute $\mathrm{CN}$. However, it should be noted that whilst the pathway is there to assist clinician evidence based decision-making, clinical discretion is still very 
much required especially with the low level of evidence that most recommendations in this pathway carry.

A number of strengths and limitations of the review need to be acknowledged. Our review was deliberately broad and, given the paucity of methodologically rigorous studies in the field, included a review of expert opinion in order to provide a comprehensive basis for development of the pathway. All identified manuscripts were reviewed for relevance and quality by at least two members of the expert panel of podiatrists. However, this was done without the use of a formal quality appraisal tool and therefore the process was not validated. High-level evidence was prioritised in the development of the pathway, however, where evidence was lacking, expert opinion was sometimes the only option. In this case, it should be acknowledged that the recommendation is based on expert opinion rather than scientific evidence and this should be reviewed as new evidence becomes available [9]. Only studies published in English between 2002-2012 were included and it is therefore possible that some relevant research was excluded. However, hand searching of reference lists, exploration of grey literature and websites, and consultation with local and international researchers is likely to have minimised this possibility.

The authors recommended that the clinical pathway be now tested for validity \& reliability, and used in larger longitudinal studies to investigate its impact on the devastating clinical outcomes of acute $\mathrm{CN}$.

\section{Conclusions}

$\mathrm{CN}$ appears to be a significantly under-recognised and under-researched complication of diabetes. Whilst $\mathrm{CN}$ remains a rare complication of diabetes, it results in significant levels of morbidity and mortality in the population of people with diabetes. Thus, immediate best practice management of this devastating complication is vital to improve clinical outcomes and patient quality of life. This systematic review, and subsequent pathway development, appears to be one of the first in the area of $\mathrm{CN}$ management. The pathway aims to support health professionals in making early diagnosis and providing appropriate immediate management of acute $\mathrm{CN}$, ultimately preventing and reducing its associated complications such as amputations and hospitalisations. It is recommended that the pathway's clinical outcomes are implemented and further researched to determine its applicability to minimise the devastating effects of $\mathrm{CN}$.

\section{Additional files}

Additional file 1: Search strategies.
Additional file 2: Level II evidence.

Additional file 3: Level III-IV evidence.

Additional file 4: Level EO evidence.

\section{Abbreviations}

CN: Charcot Neuro-Arthropathy; RCT: Randomised control trial;

NHMRC: National health and medical research council; TCC: Total contact cast; iTCC: Instant total contact cast; MRI: Magnetic Resonance Imaging;

FDG-PET: 18 F-Fluorodeoxyglucose positron emission tomography;

WCC: Leukocytosis; CRP: C-Reactive protein; ESR: Erythrocyte sedimentation rate; HbA1c: Glycosylated haemoglobin; DVT: Deep vein thrombosis; CROW: Charcot Restraint Orthotic Walker.

\section{Competing interests}

The authors have no relevant conflict of interest to disclose.

\section{Authors' contributions}

TM conceived, designed, performed literature search, quality assessments, contributed to discussion, wrote and reviewed/edited the manuscript. JR conceived, designed, performed quality assessments, contributed to the discussion, and reviewed/edited the manuscript. EK, HM, PL, TQ performed quality assessments, contributed to discussion and reviewed/edited the manuscript. FB designed, contributed to discussion and reviewed/edited the manuscript. All authors have read and approved the final manuscript.

\section{Acknowledgments}

The authors would like to acknowledge the contributions made to this project by the Podiatry Department at the Launceston General Hospital, Tasmania.

\section{Author details}

${ }^{1}$ Podiatry Department, Ipswich General Hospital, Brisbane, Australia. ${ }^{2}$ Podiatry Department, Launceston General Hospital, Launceston, Australia. ${ }^{3}$ Podiatry Department, The Prince Charles Hospital, Brisbane, Australia. ${ }^{4}$ Allied Health Research Collaborative, The Prince Charles Hospital, Brisbane, Australia. ${ }^{5}$ School of Clinical Sciences, Queensland University of Technology, Brisbane, Australia. ${ }^{6}$ Prosthetics, Orthotics and Podiatry Department, Princess Alexandra Hospital, Brisbane, Australia. ${ }^{7}$ School of Population Health, University of Queensland, Brisbane, Australia.

Received: 17 April 2013 Accepted: 24 July 2013

Published: 30 July 2013

\section{References}

1. Rogers $L C$, Frykberg RG, Armstrong DG, Boulton AJM, Edmonds M, Van $\mathrm{Ha}$ G, Hartemann A, Game F, Jeffcoate W, Jirkovska A, Jude E, Morbach S, Morrison WB, Pinzur M, Pitocco D, Sanders L, Wukich DK, Uccioli L: The Charcot Foot in Diabetes. Diabetes Care 2011, 34(9):2123-2129.

2. Frykberg RG, Zgonis T, Armstrong DG, Driver VR, Giurini JM, Kravitz SR, Landsman AS, Lavery LA, Moore C, Schuberth JM, Wukich DK, Anderson C, Vanore JV: Diabetic foot disorders: a clinical practice guideline. J Foot Ankle Surg 2006, 45(5):S1-S66.

3. Game FL, Catlow R, Jones R, Edmonds ME, Jude EB, Rayman G, Jeffcoate WJ: Audit of acute Charcot's disease in the UK: the CDUK study. Diabetologia 2012, 55:32-35.

4. Wukich DK, Sung W, Wipf, SAM, Armstrong DG: The consequences of complacency: managing the effects of unrecognized Charcot feet. Diabet Med 2011, 28:195-198.

5. Lowery NJ, Woods JB, Armstrong DG, Wukich DK: Surgical Management of Charcot Neuroarthropathy of the Foot and Ankle: a systematic review. Foot Ankle Int 2012, 33(2):113-121.

6. Richard JL, Almasri M, Schuldiner S: Treatment of acute Charcot foot with bisphosphonates: a systematic review of the literature. Diabetologia 2012, 55:1258-1264.

7. Frykberg RG, Belczyk R: Epidemiology of the Charcot Foot. Clin Podiatr Med Surg 2008, 25:17-28.

8. Leung $H B$, Ho YC, Wong WC: Charcot foot in Hong Kong Chinese diabetic population. Hong Kong Med J 2009, 15(3):191-195. 
9. National Evidence-Based Guideline on Prevention: Identification and Management of Foot Complications in Diabetes (Part of the Guidelines on Management of Type 2 Diabetes). Melbourne Australia; 2011.

10. NHMRC: A guide to the development, implementation and evaluation of clinical practice guidelines. Australia: National Health and Medical Research Council; 1999.

11. Wraight PR, Lawrence SM, Campbell DA, Colman PG: Creation of a multidisciplinary, evidence based, clinical guideline for the assessment, investigation and management of acute diabetes related foot complications. Diabet Med 2004, 22:127-136.

12. Baglioni P, Malik M, Okosieme OE: Acute Charcot foot. BMJ 2012, 344(1397):1-4

13. Pakarinen TK, Laine HJ, Maenpaa H, Mattila P, Lahtela J: The Effect of Zoledronic Acid on the Clinical Resolution of Charcot Neuroarthropathy. Diabetes Care 2011, 34:1514-1516.

14. Botek G, Anderson MA, Taylor R: Charcot neuroarthropathy: an often overlooked complication of diabetes. Cleve Clin J Med 2010, 77(9):593-599.

15. Petrova NL, Edmonds ME: Charcot neuro-osteoarthropathy - current standards. Diabetes Metab Res Rev 2008, 24(Suppl 1):58-61.

16. Rogers LC, Bevilacqua NJ: The Diagnosis of Charcot foot. Clin Podiatr Med Surg 2008, 25:43-51

17. Chantelau $\mathrm{E}$ : The perils of procrastination: effects of early vs. delayed detection and treatment of incipient Charcot fracture. Diabet Med 2005, 22:1707-1712

18. Mclntosh A, Peters J, Young R, Hutchinson A, Chiverton R, Clarkson S, Foster A, Gadsby R, O'Connor M, Rayman G, Feder G, Home PD: Prevention and Management of Foot Problems in Type 2 diabetes: clinical guidelines and evidence. Sheffield: University of Sheffield; 2003.

19. Jeffcoate WJ: Charcot neuro-osteoarthropathy. Diabetes Metab Res Rev 2008, 24(Suppl 1):62-65.

20. Chantelau E, Kimmerle R, Poll LW: Nonoperative treatment of neuroosteoarthropathy of the foot: do we need new criteria? Clin Podiatr Med Surg 2007, 24:483-503.

21. Pakarinen TK, Laine HJ, Honkonen SE, Peltonen J, Oksala H, Lahtela J: Charcot arthropathy of the diabetic foot.: current concepts and review of 36 cases. Scand J Surg 2002, 91:195-201.

22. Foltz KD, Fallat LM, Schwartz S: Usefulness of a Brief Assessment Battery for Early Detection of Charcot Foot Deformity in Patients With Diabetes. J Foot Ankle Surg 2004, 43(2):87-92.

23. Aragon-Sanchez J, Lazaro-Martinez L, Hernandez-Herrero MJ: Triggering mechanisms of neuroarthropathy following conservative surgery for osteomyelitis. Diabet Med 2010, 27:844-847.

24. Ndip A, Jude EB, Whitehouse R, Prescott M, Boulton AJ: Charcot neuroarthropathy triggered by osteomyelitis and/or surgery. Diabet Med 2008, 25:1469-1472

25. Zgonis T, Stapleton JJ, Shibuya N, Roukis TS: Surgically induced Charcot neuroarthropathy following partial forefoot amputation in diabetes. J Wound Care 2007, 16(2):57-59.

26. Bitsch M, Saunte DM, Dall C, Holstein PE: Charcot's arthropathy following digital amputation in the diabetic foot. Foot Ankle Surg 2003, 9:217-220.

27. Moura-Neto A, Fernandes TD, Zantut-Wittmann DE, Trevisan RO, Sakaki MH, Santos ALG, Nery M, Parisi MCR: Charcot foot: skin temperature as a good clinical parameter for predicting disease outcome. Diabetes Res Clin Pract 2012, 96:11-14.

28. Samann A, Pofahl S, Lehmann T, Voigt B, Victor S, Moller F, Muller UA, Wolf $\mathrm{G}$ : Diabetic Nephropathy but not $\mathrm{HbA} 1 \mathrm{c}$ is Predictive for Frequent Complications of Charcot Feet - Long-term follow-up of 164 Consecutive Patients with 195 Acute Charcot Feet. Exp Clin Endocrinol Diabetes 2012, 120:335-339.

29. Christensen TM, Bulow J, Simonsen L, Holsetin PE, Svendsen OL: Bone mineral density in diabetes mellitus patients with and without a Charcot foot. Clin Physiol Funct Imaging 2010, 30:130-134.

30. Pakarinen TK, Laine HJ, Maenpaa H, Mattila $P$, Lahtela J: Long-term outcome and quality of life in patients with Charcot foot. Foot Ankle Surg 2009, 15:187-191.

31. Christensen TM, Gade-Rasmussen B, Pedersen LW, Hommel E, Holstein PE, Svendsen OL: Duration of off-loading and recurrence rate in Charcot osteo-arthropathy treated with less restrictive regimen with removable walker. J Diabetes Complications 2012, 5:1-5.

32. Sinacore DR, Hastings MK, Bohnert KL, Fielder FA, Villareal DT, Blair VP, Johnson JE: Inflammatory Osteolysis in Diabetic Neuropathic (Charcot) Arthropathies of the Foot. Phys Therap 2008, 88(11):1399-1407.
33. Bramham $R$, Wraight $P$, May $K$ : Management of Charcot neuroarthropathy. Diabet Foot J 2011, 14(4):163-170.

34. Nube VL, McGrill M, Molyneaux L, Yue DK: From Acute to Chronic: monitoring the progress of Charcot's Arthropathy. J Am Podiatr Med Assoc 2002, 92(7):384-389.

35. Petrova NL, Moniz C, Elias DA, Buxton-Thomas M, Bates M, Edmonds ME: Is there a Systemic Inflammatory Response in the Acute Charcot Foot? Diabetes Care 2007, 30(4):997-998.

36. Petrova NL, Foster AVM, Edmonds ME: Calcaneal bone mineral density in patients with Charcot neuropathic osteoarthropathy: differences between Type 1 and Type 2 diabetes. Diabet Med 2005, 22:756-761.

37. Hastings MK, Sinacore DR, Fielder FA, Johnson JE: Bone Mineral Density During Total Contact Cast Immobilization for a Patient With Neuropathic (Charcot) Arthropathy. Phys Therap 2005, 85(3):249-256.

38. Perrin BM, Gardner MJ, Suhaimi A, Murphy D: Charcot osteoarthropathy of the foot. Aust Fam Physician 2010, 39(3):117-119.

39. Wukich DK, Sung W: Charcot arthropathy of the foot and ankle: modern concepts and management review. J Diabetes Complications 2009, 23:409426.

40. Ulbrecht JS, Wukich DK: The Charcot Foot: medical and surgical therapy. Curr Diab Rep 2008, 8:444-451.

41. Jostel A, Jude EB: Medical Treatment of Charcot Neuroosteoarthropathy. Clin Podiatr Med Surg 2008, 25:63-69.

42. De Souza L: Charcot Arthropathy and Immobilization in a WeightBearing Total Contact Cast. J Bone Joint Surg 2008, 90(4):754-759.

43. Vella S, Cachia MJ: Charcot neuroarthropathy: pathogenesis diagnosis and medical management. Malta Med J 2008, 20(3):13-19.

44. Pinzur MS, Lio T, Posner M: Treatment of Eichenholtz Stage I Charcot Foot Arthropathy with a Weight-bearing Total Contact Cast. Foot Ankle Int 2006, 27(5):324-329.

45. Pinzur MS: Current Concepts Review: Charcot arthropathy of the Foot and Ankle. Foot Ankle Int 2007, 28(8):952-959.

46. Crews RT, Wrobel JS: Physical Management of the Charcot Foot. Clin Podiatr Med Surg 2008, 25:71-79.

47. Verity S, Sochocki M, Embril JM, Trepman E: Treatment of Charcot foot and ankle with a prefabricated removable walker brace and custom insole. Foot Ankle Surg 2008, 14:26-31.

48. Hunter A: The treatment of Charcot neuroarthropathy with an Aircast Pneumatic Walker: a case study. Podiatry Now 2006, 9(7):44-47.

49. Rogers LC, Bevilacqua NJ: Imaging of the Charcot Foot. Clin Podiatr Med Surg 2008, 25:263-274.

50. Anderson LB, DiPreta J: Charcot of the Calcaneous. Foot Ankle Clin 2006 11:825-835.

51. Zampa V, Bargellini I, Rizzo L, Turini F, Ortori S, Piaggesi A, Bartolozzi C: Role of Dynamic MRI in follow-up of acute Charcot foot in patients with diabetes mellitus. Skeletal Radiol 2011, 40:991-999.

52. Schlossbauer T, Mioc T, Sommerey S, Kessler SB, Reiser MF, Pfeifer KJ: Magnetic Resonance Imaging in Early Stage Charcot Arthropathy correlation of imaging findings and clinical symptoms. Eur J Med Res 2008, 13:409-414.

53. Tan $\mathrm{PL}$, Teh J: MRI of the diabetic foot: differentiation of infection from neuropathic change. Br J Radiol 2007, 80:939-948.

54. Basu S, Chryssikos T, Moghadam-Kia S, Zhuang H, Torigian DA, Alavi A: FDG PET and PET/CT imaging in complicated diabetic foot. Semin NuCl Med 2009, 39:36-51.

55. Pickwell KM, Van Kroonenburgh MJ, Weijers RE, Van Hirtum PV, Huijberts MS, Schaper NC: F-18 FDG PET/CT Scanning in Charcot Disease: a brief report. Clin Nucl Med 2011, 36(1):8-10.

56. Basu S, Chryssikos T, Houseni M, Malay DS, Shah J, Zhuang H, Alavi A: Potential role of FDG PET in the setting of diabetic neuroosteoarthropathy: can it differentiate uncomplicated Charcot's neuroarthropathy from osteomyelitis and soft-tissue infection? Nucl Med Commun 2007, 28:465-472

57. Campanaro NR, Gurr JM, Murray RJ: Percutaneous Bone Biopsy to Distinguish Osteomyelitis from Charcot Osteoarthropathy: two case reports. Foot Ankle Int 2009, 30(12):1219-1224.

58. Johnsen B: Acute Charcot's arthropathy: a difficult diagnosis. JAAPA 2007, 20(7):22-26.

59. Judge MS: Infection and Neuroarthropathy: the utility of C-reactive protein as a screening tool in the Charcot foot. J Am Podiatr Med AssoC 2008, 98(1):1-6. 
60. Ore HM, Watkinson D: Case study: Charcot's neuropathy. Podiatry Now 2007, 10(7):25-26.

61. Stuck RM, Sohn MW, Budiman E, Lee TA, Weiss KB: Charcot Arthropathy Risk Elevation in the Obese Diabetic Population. Am J Med 2008, 121(11):1009-1014.

62. Pappalardo J, Fitzgerald R: Utilization of Advanced Modalities in the Management of Diabetic Charcot Neuroarthropathy. Am J Med 2010, 4(5):1114-1120.

63. Kennel KA, Drake MT: Adverse Effects of Bisphosphonates: implications for osteoporosis management. Mayo Clin Proc 2009, 84(7):632-638.

64. Dutta P, Bhansali A, Singh P: Charcot's Foot: advanced manifestation of diabetic neuropathy. Postgrad Med J 2004, 80:434.

65. Wunschel M, Wulker N, Gesicki M: Charcot Arthropathy of the First Metatarsophalangeal Joint. J Am Podiatr Med Assoc 2012, 102(2):161-164

66. Bernstein B, Motko J: Developing a Comprehensive Diagnostic and Treatment Plan for Charcot Neuroarthropathy - Pt. 1. Podiatry Management 2008, 2:151-161.

67. Rabinovich A: Conservative treatment can be efficacious in treatment of Charcot arthropathy. Orthopaedics Today 2010, 11:56-58.

68. Van der Ven A, Chapman CB, Bowker JH: Charcot Neuroarthropathy of the Foot and Ankle. J Am Acad Orthop Surg 2009, 17:562-571.

69. Salgami EV, Bowling FL, Whitehouse RW, Boulton AJM: Charcot Neuroarthropathy: an unusual case and a review of the literature. Int J Low Extrem Wounds 2006, 5(3):207-212

70. Yu GV, Hudson JR: Evaluation and Treatment of Stage 0 Charcot's Neuroarthropathy of the Foot and Ankle. J Am Podiatr Med Assoc 2002, 92(4):210-220.

71. Saltzman CL, Hagy ML, Zimmerman B, Estin M, Cooper R: How effective is intensive non-operative initial treatment of patients with diabetes and Charcot arthropathy of the feet? Clin Orthop Relat Res 2005, 435:185-190

72. Smith C, Kumar S, Causby R: The effectiveness of non-surgical interventions in the treatment of Charcot foot. Int J Evid Based Healthc 2007, 5:437-449.

73. Nielson DL, Armstrong DG: The Natural History of Charcot's Neuroarthropathy. Clin Podiatr Med Surg 2008, 25:53-62.

74. Bem R, Jirkovska A, Fejfarova V, Skibova J, Jude EB: Intranasal calcitonin in the treatment of acute Charcot neuroosteoarthropathy: a randomized controlled trial. Diabetes Care 2006, 29(6):1392-1394.

75. Pitocco D, Ruotolo V, Caputo S, Mancini L, Collina CM, Manto A, Caradonna $P$, Ghirlanda G: Six-month treatment with alendronate in acute Charcot neuroarthropathy: a randomized controlled trial. Diabetes Care 2005, 28(5):1214-1215

76. Yaboln CM, Duggal N, Wu JS, Shetty SK, Dawson F, Hochman MG: A Review of Charcot Neuroarthropathy of the Midfoot and Hindfoot: every radiologist needs to know. Curr Probl Diagn Radiol 2010, 39:187-199.

77. Bevan WPC, Tomlinson MPW: Radiographic Measures as a Predictor of Ulcer Formation in Diabetic Charcot MidFoot. Foot Ankle Int 2008, 29(6):568-573.

78. Ramanujam CL, Facaros Z: An overview of conservative treatment options for diabetic Charcot foot neuroarthropathy. Diabet Foot Ankle 2011, 2:1-5.

79. Rudrappa S, Game F, Jeffcoate W: Recurrence of the acute Charcot foot in diabetes. Diabet Med 2012, 29:819-821.

80. CD-ROM: International Working Group on the Diabetic Foot: international consensus on the diabetic foot. Noordwijkerhout, Netherlands: International Diabetes Federation; 2011.

doi:10.1186/1757-1146-6-30

Cite this article as: Milne et al: Developing an evidence-based clinical pathway for the assessment, diagnosis and management of acute Charcot Neuro-Arthropathy: a systematic review. Journal of Foot and Ankle Research 2013 6:30.

\section{Submit your next manuscript to BioMed Central and take full advantage of:}

- Convenient online submission

- Thorough peer review

- No space constraints or color figure charges

- Immediate publication on acceptance

- Inclusion in PubMed, CAS, Scopus and Google Scholar

- Research which is freely available for redistribution

Submit your manuscript at www.biomedcentral.com/submit
Ciomed Central 